\title{
Research on the Construction of Dongying Wetland City during the "14th Five-Year Plan"
}

\author{
Jiaming Wang ${ }^{1 *}$, Yunfei Zhang ${ }^{1}$, and Xueyi $\mathrm{Du}^{1}$ \\ ${ }^{1}$ School of Chinese Law and Economics Management, Shengli College China University of Petroleum, Dongying, China
}

\begin{abstract}
The 18th National Congress of the Communist Party of China proposed to place ecological civilization construction in the important position of "five in one", and with the transformation of economic development mode, focusing on high-quality, scientific, and reasonable urban development model has become the research focus of experts and scholars, with certain theories And practical significance. Taking the opportunity of Dongying being rated as an "International Wetland City", this article studies the construction of Dongying Wetland City during the "14th Five-Year Plan" period. Starting from the investigation of the current situation, it analyzes the current situation of Dongying City's wetland and puts forward the problems in the construction of Dongying City's wetland city. Combing the experience of Haikou, Berlin, Freiburg, and Vienna experience in wetland city construction at home and abroad, and analyzed the wetland foundation from the four perspectives of wetland protection, wetland restoration, wetland utilization, and wetland management, and proposed Dongying The city's policy recommendations for wetland construction are designed to provide suggestions for the construction of Dongying wetland city during the "14th Five-Year Plan" period, to provide a reference for the same type of regions, and to provide a reference for the research of experts and scholars in the same field.
\end{abstract}

\section{Introduction}

The report of the 19th National Congress of the Communist Party of China clearly stated that it is necessary to accelerate the construction of ecological civilization, advance system reforms, and build a beautiful new China, and continue to increase the protection of natural ecosystems and strengthen the protection and restoration of wetlands; Shandong Provincial People's Government Order Wetland protection in Shandong Province has been clearly stipulated in terms of a wetland nature reserve and the wetland park construction, wetland protection planning, wetland supervision and management, and legal responsibilities for wetland damage; on October 29, 2018, Dongying City was approved by the world In the first batch of international wetland cities, the municipal party committee and municipal government have made the construction of international wetland cities a key task; At the beginning of the establishment of Dongying City, the city was positioned as a "wetland city, the ecological city". The Fourth Plenary Session of the Sixth Municipal Party Committee put forward the proposal. In order to create the urban characteristics of "wetland in the city, city in the wetland", to create a high-quality development growth pole in Shandong, the Yellow River into the sea cultural tourism destination, and build a modern wetland city full of vitality. It can be seen from the successive policies issued by the above countries and Shandong Province: the state, Shandong Province,
Dongying City and other levels all provide policy support for wetland protection and modern wetland cities. It is imperative for Dongying to build a modern wetland city; at the same time, Dongying City As a traditional resource city, how to transform in the later stage of resource development is an urgent problem to be solved. Natural wetland conditions can be used as a breakthrough point in urban transformation, and can also contribute to the construction of a modern wetland city, an eco-tourism city, and a national civilized city in Dongying. contribution. 2020 is the final year of the "13th Five-Year Plan". Given that domestic and foreign wetland cities, wetland tourism and other wetland protection and development experience are less and less useful for reference, how about Dongying Wetland City during the "14th Five-Year Plan" Construction, how to sort out the path of modern wetland city construction at home and abroad, identify the targets for reference, choose the direction of reference, how to combine the conditions of Dongying city and the specific conditions of wetlands to carry out sustainable development and explore the development path under the premise of protection. Focus.[1]

Taking the opportunity of Dongying City being rated as an "International Wetland City", this research discusses the construction of Dongying Wetland City during the "14th Five-Year Plan" period. Starting from the investigation of the current situation, it analyzes the current situation of Dongying City's wetland and proposes the existence of wetland city construction in

\footnotetext{
* Corresponding author: upcjgyxwjm@163.com
} 
Dongying Questions, sorted out the Haikou experience, Berlin experience, Freiburg experience, and Vienna experience for reference in wetland city construction at home and abroad, and analyzed the wetland foundation from four perspectives: wetland protection, wetland restoration, wetland utilization, and wetland management. Administrative guarantees, institutional guarantees, exchanges and cooperation, rural revitalization, industrial structure optimization, transportation optimization, space planning, garbage disposal, and wetland urban culture put forward suggestions for the construction of Dongying wetland city, aiming to provide Dongying wetland during the "14th Five-Year Plan" period. Suggestions on urban construction provide references for the same types of regions and the research of experts and scholars in the same field.

\section{Status and Problems of Wetland and Wetland City Construction in Dongying City}

From the "five-in-one" overall layout of the 18th National Congress of the Communist Party of China to the concept of green development, from the 19th National Congress of the Communist Party of China to "accelerate the reform of the ecological civilization system and build a beautiful China", to Xi Jinping's thoughts on ecological civilization, etc., this series of new Concepts, new thoughts and new strategies have profoundly pointed out the extreme importance of ecological environment construction and demonstrated the country's firm determination to the construction of ecological civilization. Provinces and cities have always attached great importance to the protection and restoration of wetlands. Dongying City has positioned the city as a "city of wetlands and an ecological city" at the beginning of its establishment. Dongying City's wetland conditions are unique and possess the natural resource endowment for the construction of a wetland city. However, with the economic development and changes in the Yellow River basin in recent years, the Yellow River Delta wetland has shown problems affecting the construction of wetland cities, mainly in the construction of wetlands and wetland cities.

Wetland issues can mainly start from aspects such as wetlands, water resources, and biodiversity. The Yellow River Delta wetland ecosystem has the characteristics of youth, fragility, and instability. The ecological environment is very fragile. Especially in the context of accelerated global climate change, once a certain condition (such as the lack of freshwater resources) changes, The wetland ecosystem will undergo reverse succession, the consequence of which is the degradation of wetland resources, and the loss of existing bird habitats and the species and number of birds will undergo rapid changes. Therefore, the most important and basic work to create a cultural tourism destination for the Yellow River to enter the sea should be the protection and restoration of the Yellow River Delta wetland ecosystem. The Yellow River Delta wetlands are formed by alluvial sedimentation of the Yellow River. The inflow of water and sediment from the upper reaches of the Yellow River decreases, resulting in a decrease in a natural wetland area, wetland shrinkage, and decline in wetland functions. The wetland ecological water demand gap is large, and the original wetland ecosystem is threatened; this is in line with the Yellow River. The upstream water and soil conservation work is closely related, so it is necessary to strengthen the overall coordinated development of the Yellow River Basin. With the over-exploitation and utilization of water resources, serious water pollution and reduced wetland biodiversity have affected the stability and sustainability of the ecosystem. In the process of urban development, oil exploration, exploration, development and other production and operation activities in Dongying City are relatively frequent, causing ecological problems such as wetland degradation in some wetlands in Dongying City. Natural wetlands have been shrinking, and a large number of scattered and broken wetlands have appeared. The wetland ecology has been severely affected.

The problem of wetland city construction is mainly from the aspect of planning and design. The existing wetland city construction of Dongying City is in its infancy. The factors of wetland city, high-quality development, and green sustainable development have been integrated into municipal planning, but at the same time, it should be better. The combination of modern Internet information technology, artificial intelligence and other aspects to build a modern smart wetland city; at the same time, in the process of wetland city construction, it is necessary to solve the original infrastructure, city service level, carrying capacity and the original ecological problems of the wetland. The high-quality construction of wetland cities.

\section{Reference from domestic and foreign experience in wetland city construction}

It summarizes the experience of wetland city construction at home and abroad, aiming to provide experience and reference for the construction of Dongying City and similar wetland cities. However, since most of the construction of wetland cities starts from urban wetland planning, green city construction, etc., this part will expand while combing wetland cities for reference and compare and analyze the experience of related urban construction, to provide a more comprehensive experience for Dongying City. Wetland city construction has made references and contributions.[2]

The research is mainly based on the domestic and foreign experience of wetland cities and green cities in Haikou, Berlin, Freiburg, Vienna and other cities. In addition, there are also water-sensitive cities in Barcelona, Amsterdam, Portland, Toronto, and Australia. Singapore's ABC planning, etc., has developed a wetland city or green city for the construction and planning of wetland cities or green cities from various aspects such as development laws and policies, humanized urban public space, technological innovation, 
public transportation, market government integration, and infrastructure construction, forming a reference for the construction and planning of wetland cities or green cities. Wetland city construction experience.[3-4]

\section{Countermeasures for Dongying Wetland City Construction during the "14th Five-Year Plan"}

From the current situation of wetland and wetland city construction in Dongying City, and the domestic and foreign experience of wetland city construction, it can be seen that the construction of wetland city in Dongying City during the "14th Five-Year Plan" period should not only feature wetland cities, but also use existing smart cities, The construction of a green city is an opportunity to correctly handle the relationship between river and wetland, green and wetland, landscape and wetland, implement water system penetration, wetland restoration, and background greening projects to build and improve the city's wetland protection system to achieve river connectivity, water connectivity, and energy circulation, To create an ecological landscape with clear rivers, clear wetlands, and beautiful shore greens, to show the unique features of Dongying City's "Hehai Lake Wetland City", plan the construction of a digital national wetland park and a digital world wetland museum, and rationally layout the eco-tourism experience area. Construct the Great River Delta Ecological Grand View Garden and create a world-renowned wetland ecosystem experience destination. On the basis of ensuring the urban ecology, population and comprehensive carrying capacity, construct a modern wetland city. It is true that the construction of wetland cities cannot be accomplished overnight, and long-term planning and implementation are required. Therefore, this part studies the countermeasures of Dongying wetland city construction during the "14th Five-Year Plan" period, mainly from the two aspects of wetland foundation and urban upgrading.

\subsection{Basic aspects of wetland}

\subsubsection{Wetland protection}

Establish a coordination mechanism for wetland protection to coordinate protection of biodiversity and natural resources such as wetlands, water, forests, fields, lakes, and grasses, form a wetland protection mechanism for the living community of wetland resources, and build wetland ecological landscape belts and ecological protection barriers. Use ecological protection measures to carry out a protective overall restoration of existing wetlands and other community resources.

\subsubsection{Wetland restoration}

First, strengthen the restoration and protection of broken and scattered wetlands. Give full play to the role of scattered wetlands in absorbing and storing floods, and lay the foundation for the later development of continuous or characteristic wetlands. Second, protect the ecological diversity of wetlands, prohibit the invasion of alien species, and protect them from a legislative perspective. Third, make breakthroughs and research and development in wetland restoration technology, and cooperate in research and development with local or regional neighboring universities, research institutes and other scientific research forces. Fourth, set up a special institution for wetland restoration and set up special funds to carry out compensation for wetland ecological benefits, and absorb multiple forces including governments, enterprises, federations, and the public to carry out wetland restoration. Fifth, draw lessons from international and domestic excellent cases and enhance the capacity and level of wetland restoration through cooperation and exchanges, so as to better lay the foundation for the construction of wetland cities.

\subsubsection{Wetland utilization}

From the wetland ecological industry, wetland ecological agriculture, wetland ecological tourism, wetland ecological service industry, etc., the industrial structure layout is carried out to make ecological industrialization, and promote the effective use of land resources and the effective play of their overall functions.

\subsubsection{Wetland management}

Give full play to modern Internet information technology, artificial intelligence, etc., further improve the level of information management and scientific research monitoring capabilities, and gradually build a modern and intelligent management system. Based on the wetland scientific research monitoring center, combined with advanced technology to construct a threedimensional and comprehensive intelligent ecological monitoring system, build a comprehensive intelligent wetland information platform to realize the integration and sharing of information and data, and at the same time make the wetland protection, restoration and utilization Technical and management guarantees.

\subsection{The improvement of wetland cities}

\subsubsection{Administrative guarantee}

Persist in the integration and coordination of "a game of chess", strengthen top-level design, overall planning, upper and lower linkages, and left and right interactions, breaking the boundaries of each county and district and each department fighting alone and fighting each other, and achieve seamless docking to ensure that no dead ends or blind spots are left. Set up a special wetland city planning bureau to coordinate and coordinate the actual basic work of wetland protection, wetland restoration, wetland utilization and wetland management and wetland city upgrading, and coordinate the coordinated progress of wetland city construction, spatial planning, 
smart city and other tasks. Promote the integration and advancement of related work, and provide administrative guarantees in the overall planning process of wetland city construction.

\subsubsection{Exchange and cooperation}

Dongying City can rely on the location advantages of the Yellow River Delta to communicate and promote with international wetland cities and various international conferences, exchange and learn new concepts and new technologies in wetland protection, restoration, utilization and management, and at the same time contribute to the construction of global wetland cities Chinese wisdom and Chinese solutions. At the same time, more domestic cities with Changde, Changshu, Harbin, Haikou, and Yinchuan have been selected as international wetland cities to establish the International Wetland City Innovation Alliance, build an organizational collaboration platform, strengthen technological and management innovation, and form a leading demonstration Effect, and contribute to the construction of their respective wetland cities and a beautiful China.

\subsubsection{Industrial structure optimization}

Taking the opportunity of the integration of the local refinery in Shandong Province, adhere to the principles of market leadership, government guidance, legal compliance, strict standards, etc., to promote the integration, industrialization, and intensification of the existing refining and chemical industries in Dongying City, so as to achieve the high-quality development of the local refinery industry while helping The construction of wetland cities.

\subsubsection{Traffic optimization}

During the construction of wetland city, Dongying City should increase the role of public transportation in citizens' travel according to local conditions. On the one hand, it can alleviate traffic congestion. On the other hand, it can reduce the emission of automobile exhaust and other pollution, and promote the improvement of the quality of the urban ecological environment. Promote the construction of wetland cities.

\subsubsection{Wetland City Culture}

We will deepen the creation of wetland city culture as the overall starting point to improve the quality of urban functions and improve the level of urban governance capabilities, as an important measure to accelerate the construction of a modern wetland city with vigor, highlight the key points, improve the mechanism, and constantly focus on unremitting efforts for a long time. Higher standards promote the creation of cities in the same direction as urban development.

\section{Conclusion}

During the "14th Five-Year Plan" period, we carried out research on the construction of Dongying wetland city. Starting from the current situation investigation, we analyzed the current situation of Dongying city wetland and put forward the problems existing in the construction of Dongying city wetland. The experience of Haikou, Berlin, Freiburg, and Vienna We will sort out the domestic and foreign experience of wetland city construction, and provide references for related research and related wetland city construction. From the aspects of wetland foundation and wetland city improvement, the countermeasures for wetland city construction in Dongying City are proposed to provide intellectual support for the construction of wetland city in Dongying City.

\section{Acknowledgment}

The Humanities and Social Science Foundation of the Ministry of Education (20YJC790132); National Statistical Science Research Project (2020LY069); Shandong Provincial Natural Science Foundation (ZR2020QG042); Shandong Province Educational Science Planning (2020ZC221); Shandong Province Humanities and Social Sciences Project (19-ZC-JJ-03); Shandong Province Art Science Key Project (ZD202008343); Key Funding Projects of Dongying City Social Science Planning (DYSK (2021) No. 2).

\section{References}

1. Lin Zhibin. Discussion on the practice of promoting urban development in Guangzhou Haizhu National Wetland Park [J]. Environmental Ecology, 2020, 2(08): 96-98.

2. Zhang Lang. Research on the Protection and Restoration of Urban Wetland Landscape[J]. Garden, 2020(08): 1 .

3. Xiao Bing. Quality control of key construction techniques for ecological restoration of urban typical artificial lake wetlands: Taking an artificial lake in Hangzhou as an example [J]. Garden, 2020(08): 2328.

4. Cao Haixia.The impact of constructed wetland on the urban ecological environment[J]. Environment and Development,2020,32(07):190-191. 